\title{
Evidence for Diquarks in Lattice QCD
}

\author{
C. Alexandrou, ${ }^{1} \mathrm{Ph}$. de Forcrand, ${ }^{2,3}$ and B. Lucini ${ }^{2,4}$ \\ ${ }^{1}$ Department of Physics, University of Cyprus, CY-1678 Nicosia, Cyprus \\ ${ }^{2}$ Institute for Theoretical Physics, ETH Zurich, CH-8093 Zurich, Switzerland \\ ${ }^{3}$ CERN, Physics Department, TH Unit, CH-1211 Geneva 23, Switzerland \\ ${ }^{4}$ Department of Physics, University of Wales Swansea, SA2 8PP Swansea, United Kingdom
}

(Received 5 September 2006; published 30 November 2006)

\begin{abstract}
Diquarks may play an important role in hadron spectroscopy, baryon decays, and color superconductivity. We investigate the existence of diquark correlations in lattice QCD by considering systematically all the lowest energy diquark channels in a color gauge-invariant setup. We measure mass differences between the various channels and show that the positive parity scalar diquark is the lightest. Quark-quark correlations inside the diquark are clearly seen in this channel, and yield a diquark size of $\mathcal{O}(1) \mathrm{fm}$.
\end{abstract}

DOI: 10.1103/PhysRevLett.97.222002

Diquarks were originally proposed several decades ago [1] as a natural way to explain the absence of a large number of exotics otherwise predicted by QCD. Recently, it has been realized that they provide a natural explanation for an exotic baryon antidecuplet, the $\Theta^{+}[2]$, that cannot be accommodated in the quark model. The first evidence for the $\Theta^{+}$was reported by the LEPS Collaboration [3], but subsequent experiments cast doubts on its existence [4]. Independently of whether a pentaquark exists or not, diquark correlations may play an important role in the description of quark distributions and fragmentation functions and in explaining the systematics of nonleptonic weak decays of light quark hadrons [5]. In addition, diquarks are the central ingredient of cold, dense matter where they condense to form a color superconductor. Despite their potential role in explaining such a variety of phenomena in hadronic physics, quantitative analyses that can directly assess whether diquark correlations are present in QCD are lacking. Lattice QCD is the method of choice for studying hadronic states. However, diquarks are colored objects and need an appropriate formalism to study them using lattice simulations. One way is to fix the gauge as done in an early study on the mass of the diquarks [6]. In this work we present a gauge-invariant formalism, where we create color singlet states by considering diquarks in the background of a static quark $[7,8]$. This enables us to extract mass differences between baryons containing diquarks in the various channels. These mass differences, unlike diquark masses themselves, are well defined and gauge invariant.

One gluon exchange indicates quark-quark attraction in the color antitriplet $\overline{3}_{c}$, flavor antisymmetric $\overline{3}_{f}$, and spin singlet positive parity channel. Diquarks in this channel are referred to as "good" diquarks [5]. The aim of this work is to check whether QCD dynamics supports attraction in this channel and compare it with other diquark channels. Possible diquark configurations are created by two quark operators and insertions of the covariant derivative. In this work we consider only diquark configurations with no derivatives because these are lower in energy. We therefore
PACS numbers: 12.38.Gc, 11.15.Ha, 12.38.Aw

consider all 16 diquark multiplets that can be created by operators bilinear in the quark fields of the form $q^{T} C \Gamma q$ with $C=i \gamma_{0} \gamma_{2}$ the charge conjugation operator, and $\Gamma=$ $1, \gamma_{\mu}, \gamma_{5}, \gamma_{5} \gamma_{\mu}, \sigma_{\mu \nu}$. The positive parity channels are $q^{T} C \gamma_{5} q$ and $q^{T} C \gamma_{5} \gamma_{0} q$ with spin zero and $q^{T} C \gamma_{i} q$ and $q^{T} C \sigma_{0 i} q$ with spin one. The negative-parity channels are $q^{T} C q$ and $q^{T} C \gamma_{0} q$ with spin zero and $q^{T} C \gamma_{5} \gamma_{i} q$ and $q^{T} C \sigma_{i j} q$ with spin one. They create states that vanish in the nonrelativistic limit and are excluded in quark models. We expect them to be higher in energy.

In addition to mass differences, we probe correlations directly by examining the spatial distribution of the two quarks forming the diquark in the background of the static quark [7]. Correlations which persist as the static quark is removed are intrinsic to the diquark. We study these correlations using gauge-invariant two-density correlators, used before [9] to probe hadron structure:

$$
C_{\Gamma}\left(\mathbf{r}_{u}, \mathbf{r}_{d}, t\right)=\left\langle 0\left|J_{\Gamma}(\mathbf{0}, 2 t) J_{0}^{u}\left(\mathbf{r}_{u}, t\right) J_{0}^{d}\left(\mathbf{r}_{d}, t\right) J_{\Gamma}^{\dagger}(\mathbf{0}, 0)\right| 0\right\rangle,
$$

where $J_{0}^{f}(\mathbf{r}, t)=: \bar{f}(\mathbf{r}, t) \gamma_{0} f(\mathbf{r}, t):, f=u, d$, and

$$
J_{\Gamma}(x)=\epsilon^{a b c}\left[u^{T}{ }_{a}(x) C \Gamma d_{b}(x) \pm d^{T}{ }_{a}(x) C \Gamma u_{b}(x)\right] s_{c}(x),
$$

where the $+(-)$ sign corresponds to the flavor symmetric (antisymmetric) combination, and $s_{c}$ denotes the static quark. Latin indices denote color. We use two degenerate flavors of Wilson fermions on quenched configurations generated at $\beta=5.8,6.0$, and 6.2 corresponding to lattice spacing $a=0.136,0.093$, and $0.068 \mathrm{fm}$ as determined from the static quark force [10]. At each lattice spacing, we perform measurements at three values of the pion mass in the range 570-910 MeV. By comparing results obtained at different lattice spacings but at the same pion mass we can assess discretization effects. In most cases the quenched approximation is good when the mass of the pion is higher than $600 \mathrm{MeV}$, but is expected to fail in the chiral limit. In order to check the validity of our results in this range of pion masses, we repeat our mass splitting 
TABLE I. Summary of our simulations, including the value of $\kappa$, which determines the bare quark mass, the pion mass, $m_{\pi}$, and nucleon mass $M_{N}$ in lattice and, for the latter, in physical units and the number of gauge configurations.

\begin{tabular}{lccc}
\hline \hline$\kappa$ & $a m_{\pi}$ & $a M_{N}\left[M_{N} \mathrm{GeV}\right]$ & Number of confs \\
\hline \multicolumn{5}{c}{ Quenched $16^{3} \times 32 \beta=5.8, a^{-1}=1.47 \mathrm{GeV}$} \\
0.1560 & $0.619(2)$ & $1.139(8)[2.04(1)]$ & 356 \\
0.1575 & $0.549(2)$ & $1.052(9)[1.55(1)]$ & 160 \\
0.1590 & $0.473(2)$ & $0.961(9)[1.41(1)]$ & 200 \\
0.153 & $0.423(1)$ & $0.783(8)[1.68(2)]$ & 364 \\
0.154 & $0.366(2)$ & $0.716(7)[1.54(2)]$ & 503 \\
0.155 & $0.300(2)$ & $0.634(8)[1.36(2)]$ & 287 \\
0.1510 & $0.286(3)$ & $0.563(8)[1.66(2)]$ & 116 \\
0.1520 & $0.214(3)$ & $0.487(9)[1.43(3)]$ & 166 \\
0.1523 & $0.188(3)$ & $0.460(11)[1.35(3)]$ & 157
\end{tabular}

Unquenched Wilson $24^{3} \times 40 \beta=5.6, a^{-1}=2.42 \mathrm{GeV}$

$0.1575 \quad 0.270(3) \quad 0.580(7)[1.40(2)]$

185

measurements on a set of configurations with two degenerate flavors of dynamical Wilson fermions at $\beta=5.6$ [11]. In Table I we collect the parameters of our calculation.

The mass of our static-light-light baryon can be obtained from the large time limit of the correlator $G_{\Gamma}(t)=$ $\left\langle J_{\Gamma}(\vec{x}, t) J_{\Gamma}^{\dagger}(\vec{x}, 0)\right\rangle$. The effective mass, $m_{\mathrm{eff}}(t) \equiv$ $-\log \left[G_{\Gamma}(t) / G_{\Gamma}(t-1)\right]$, becomes time independent for large $t$ when the lightest state dominates (plateau region), yielding its mass. To reduce the statistical errors, we need to keep $t$ small by isolating the ground state as fast as possible. This is accomplished by suppressing higher excitations using Wuppertal smearing [12] on the source and sink with hypercubic (HYP) smeared spatial links [13] for the Wuppertal smearing function [14]. In addition, we use HYP smearing on the temporal gauge links $U_{4}(x)$ that enter in the construction of the static propagator given by

$$
S_{\text {stat }}\left(\mathbf{x}_{2}, t_{2}, \mathbf{x}_{1}, t_{1}\right)=\delta^{3}\left(\mathbf{x}_{2}-\mathbf{x}_{1}\right)\left(\frac{1+\gamma_{0}}{2}\right)\left[\prod_{t=t_{1}}^{t_{2}-a} U_{4}\left(\mathbf{x}_{1}, t\right)\right]^{\dagger}
$$

for $t_{2}>t_{1}$. The exponential prefactor $\exp \left[-m_{q}\left(t_{2}-t_{1}\right)\right]$ has been dropped, because this introduces a constant shift in all energies by the bare heavy quark mass $m_{q}$, which cancels in the mass differences that we measure between baryons with diquarks in different channels.

We show in Fig. 1 the effective mass differences $\Delta m_{\text {eff }}$ between the good (scalar) diquark created by taking $\Gamma=$ $\gamma_{5}$ and the other channels. As expected the mass difference is zero for the other scalar diquark with positive parity created by taking $\Gamma=\gamma_{0} \gamma_{5}$. The other positive parity channels, i.e., the "bad" (vector) diquarks, are degenerate and are clearly higher in energy than the good diquarks as

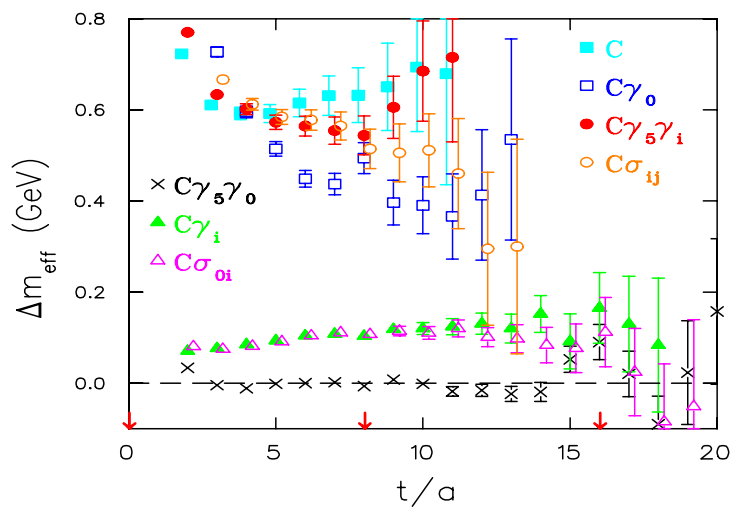

FIG. 1 (color online). Effective mass difference $\Delta m_{\mathrm{eff}}$ in the various diquark channels, at $\beta=6.0$ for our lightest quarks. The arrows (from left to right) indicate the time slice of the source, of the density insertions, and of the sink.

pointed out in Ref. [5]. The negative-parity states, as expected, have even larger energies. Their effective masses are much noisier making it difficult to identify a clear plateau void of contamination from yet higher states. The general behavior is, however, similar to the positive parity diquarks: the vector channels are also degenerate and tend to have a larger mass than the scalar channel created by taking $\Gamma=I$. To verify that the mass difference seen between the two scalar channels is significant one would need to improve the quality of the mass plateaus.

Effective color-spin Hamiltonian arguments [5] predict that for heavy constituent quarks the mass difference $\Delta m \equiv m_{\gamma_{i}}-m_{\gamma_{5}}$ between the good and bad diquarks scales like $1 / m_{q_{1}} m_{q_{2}}$, where $m_{q_{1,2}}$ are the masses of the constituent quarks. On the other hand, in the light quark regime we have $m_{\pi}^{2} \propto m_{q}$. Therefore for intermediate values of $m_{q}$ where both relations hold approximately we make the ansatz $\Delta m \propto 1 / m_{\pi}^{4}$. In the opposite limit $m_{q} \rightarrow$ $0, \Delta m$ is expected to approach a constant value. These two behaviors can be connected by the ansatz

$$
\Delta m=\frac{c_{1}}{1+\left(m_{\pi} / c_{2}\right)^{4}},
$$

with $c_{1}$ and $c_{2}$ to be determined from the lattice data. We show in Fig. 2 results for $\Delta m$ as a function of $m_{\pi}^{4}$ for our three $\beta$ values. All lattice data should fall on a universal line if we are close to the continuum limit. We observe that this is indeed the case on our two finest lattices whereas for the coarsest, scaling violations are apparent. In the same figure we also show $\Delta m$ obtained using unquenched, dynamical Wilson configurations. It nicely falls on the same curve as the fine-lattice quenched results. This corroborates that scaling has set in and that quenching effects at these quark masses are small. Therefore a quenched study of diquark properties in the quark mass range used here is a very good approximation and will be adopted in the rest of the discussion. The ansatz given in Eq. (4) provides a good 


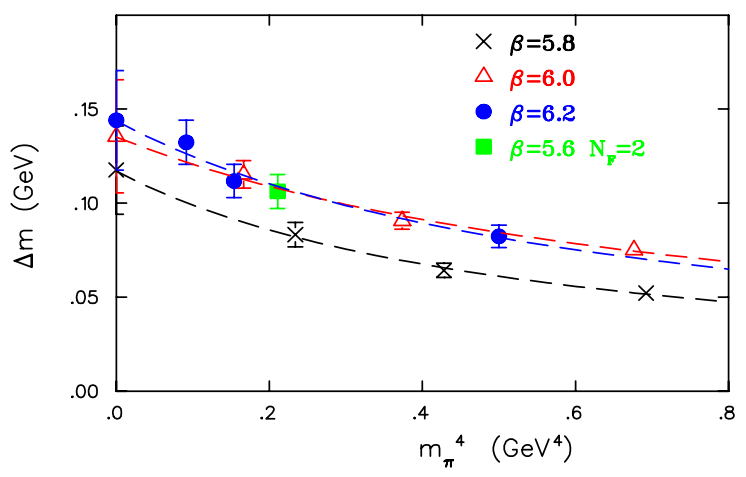

FIG. 2 (color online). Mass difference between good and bad diquarks as a function of $m_{\pi}^{4}$ at $\beta=5.8$ (crosses), $\beta=6.0$ (open triangles), $\beta=6.2$ (solid circles), and unquenched result (solid square). The dashed lines are fits to Eq. (4).

fit to the lattice data. Using the lattice data with good scaling behavior at $\beta=6.0$ and 6.2 , we find $c_{2}=0.78 \pm$ $0.15 \mathrm{GeV}$, and $c_{1}=0.138 \pm 0.01 \mathrm{GeV}$, which is the mass difference at the chiral limit [15]. This mass splitting can be compared to the $\Delta$-nucleon mass splitting, $\delta m_{\Delta N}$. Using our quenched data at $\beta=6.0$ we find for the ratio $\Delta m / \delta m_{\Delta N}=0.67(7), 0.73(8)$, and 0.67(8) at $\kappa=0.153$, 0.154 , and 0.155 , respectively. Therefore our estimate of $\Delta m$ is consistent with the predicted $\frac{2}{3}$ of the $\Delta$-nucleon mass difference as given in Ref. [5].

Having determined the diquark spectrum, we now turn to the analysis of their structure by studying the densitydensity correlators defined in Eq. (1). The time $t$ where the density operators are inserted is shown in Fig. 1 by the arrow (at $t / a=8$ ), and is within the plateau range of the effective mass. We have verified that the correlators remain, within errors, unchanged when we vary the sourcesink separation or equivalently $t$. Our aim is to look for spatial correlations between the two light quarks in the various diquark channels. We take the location of the static quark as the reference coordinate from which the distances $\mathbf{r}_{u}$ and $\mathbf{r}_{d}$ of the two quarks of different flavors are measured. We are interested in intrinsic diquark correlations, which persist as the static quark is moved away from the diquark. We therefore consider spherical shells $\left|\mathbf{r}_{u}\right|=$ $\left|\mathbf{r}_{d}\right|=r$ of increasing radius $r$. Because the system is spherically symmetric the correlator depends only on $r$ and the angle $\theta=\arccos \left(\hat{\mathbf{r}}_{u} \cdot \hat{\mathbf{r}}_{d}\right)$ [7]. In the absence of any correlation, the distribution of $C_{\Gamma}(r, \theta)$ will be uniform as a function of $\cos (\theta)$. Attraction will show up as an enhancement at small angles, near $\cos (\theta)=1$. Our cubic lattice breaks rotational symmetry and distorts the uniform spherical distribution, particularly at small angle $\theta$. To remove such lattice artifacts, we normalize our distributions by a uniform lattice distribution. We show the resulting density correlators in Figs. 3 and 4 for various shell radii $r$. The physically relevant correlations are those that survive when $r$ is large. We indeed observe that the qualitative behavior of the distributions does not depend on $r$, confirming that the color field generated by the static quark does not affect the physics of diquarks once $r$ is large enough.

In Fig. 3 we show $C_{\Gamma}(r, \theta)$ for all the different channels as a function of $\cos (\theta)$ when the shell radius is fixed to $r=$ $0.5 \mathrm{fm}(r / a=7.35)$. We clearly observe that the good diquark shows enhanced correlations at small $\theta$, indicating attraction between the quarks. There is also a gradual increase in the distribution for the bad diquark indicating a weaker attraction in this channel. This behavior persists as we increase or decrease the quark masses. For the negative-parity channels, correlations are absent or very weak, except for the vector channel with $\Gamma=\gamma_{5} \gamma_{i}$. However, those disappear for lighter quark masses. The negative-parity correlators are noisier because the diquarks are heavier, so that a higher statistics analysis will be needed to fully determine the characteristics of their distributions. For this reason, we now restrict our analysis to the positive parity diquarks.

In Fig. 4 we show the density correlators for the good (scalar) and bad (vector) diquarks for all three lattice spacings at our lightest quark mass. The left hand set shows the correlator as a function of $\cos (\theta)$ normalized to 1 at $\theta=0$ for $r / a=5.1$, which corresponds to $r=0.69,0.47$, and $0.35 \mathrm{fm}$ at $\beta=5.8,6.0$, and 6.2 , respectively. As can be seen, irrespectively of the distance the good diquark shows stronger spatial correlations. Within this framework we can also extract the diquark size: At fixed $r$ we look at the dependence of the correlator on the relative $u$ - $d$ separation, $r_{u d}=2 r \sin (\theta / 2)$. In the right hand of Fig. 4, we show the correlator (normalized to 1 at the origin) as a function of $r_{u d}$ for a fixed physical shell radius $r=0.5 \mathrm{fm}$, at our lightest quark mass. An exponential dependence of $C_{\gamma_{5}}\left(r, r_{u d}\right) \propto \exp \left[-r_{u d} / r_{0}(r)\right]$ provides a gauge-invariant definition of the diquark size $r_{0}(r)$ for a given value of $r$. The curves in Fig. 4, obtained from fits to an exponential dependence, describe well $C_{\gamma_{5}}\left(r, r_{u d}\right)$ at all $\beta$ values.

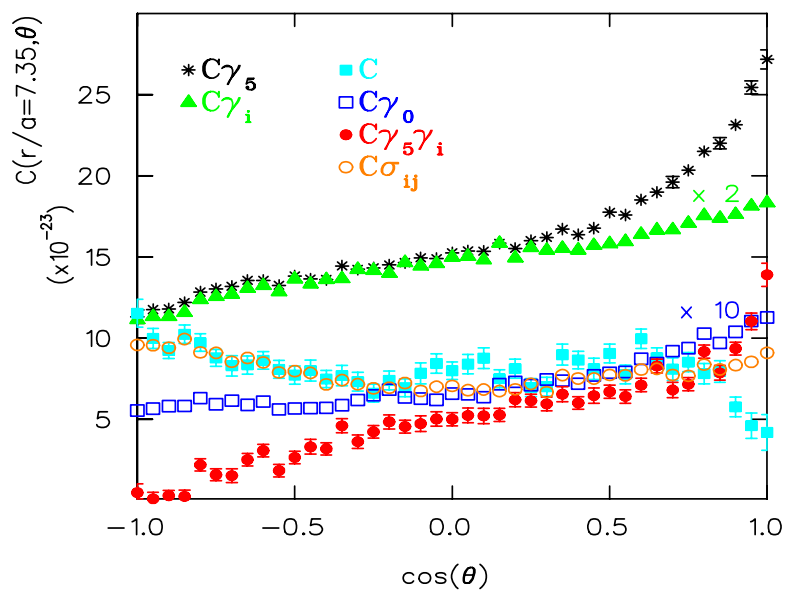

FIG. 3 (color online). $\quad C_{\Gamma}(r / a=7.35, \theta)$ versus $\cos (\theta)$ for $\beta=$ 6.2 and $\kappa=0.1520$ for the good diquark (asterisks) and the other diquark channels using the same notation as in Fig. 1. Correlators for the bad diquark have been multiplied by two and for all negative-parity channels by ten. 


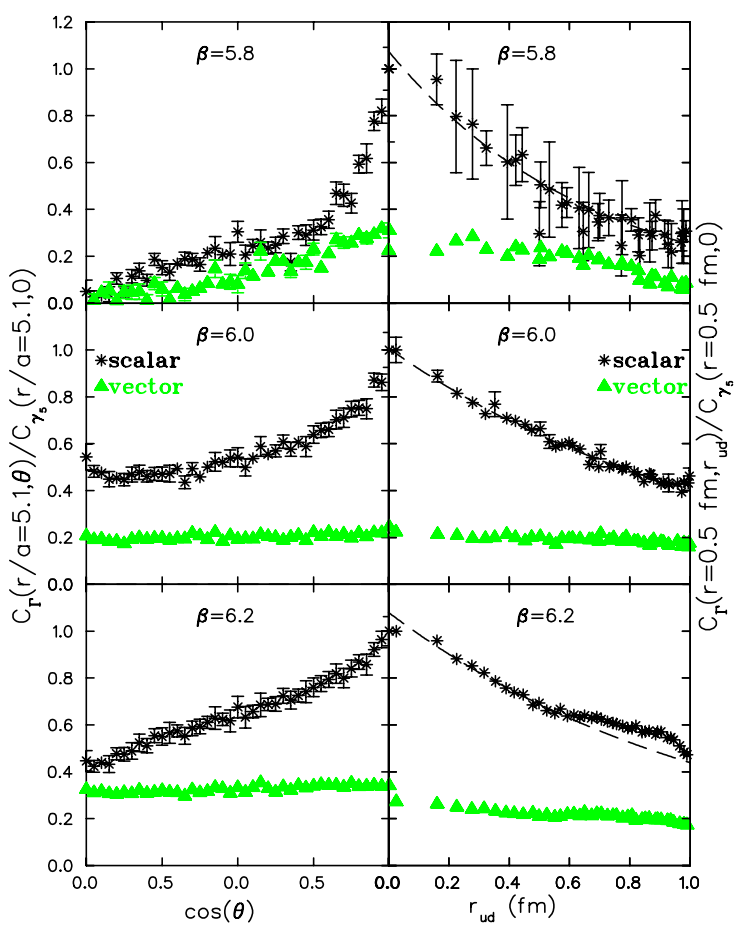

FIG. 4 (color online). Left: $C_{\Gamma}(r / a=5.1, \theta) / C_{\gamma_{5}}(r / a=5.1,0)$ versus $\cos (\theta)$. Right: $C_{\Gamma}\left(r=0.5 \mathrm{fm}, r_{u d}\right) / C_{\gamma_{5}}(r=0.5 \mathrm{fm}, 0)$ versus $r_{u d}$, for the good (asterisks) and bad (solid triangles) diquarks at the lightest pion for our three lattice spacings.

Moreover, the physical size $r_{0}(r)$ is the same on the two finer lattices, confirming continuumlike behavior as for the diquark masses. We can then evaluate $r_{0}$ as a function of the shell radius $r$. Our measurements show a mild increase of $r_{0}$ with $r$, from $\sim 0.9 \mathrm{fm}$ to $\sim 1.3 \mathrm{fm}$ as $r$ increases from $0.3 \mathrm{fm}$ to $0.75 \mathrm{fm}$. For large $r \geq 0.5 \mathrm{fm}, r_{0}$ is consistent with having reached a plateau. This suggests that the static source has a small influence on the good diquark giving a characteristic size of about $1.1 \pm 0.2 \mathrm{fm}$. For comparison, at the same quark mass and using the same definition of size, we find for the $\rho$ a size of $0.7 \mathrm{fm}$ [9]. The large diquark size can be understood from the following qualitative argument: As the static quark is moved away from the diquark a $q-q$ string tension develops. Considering an increase $\epsilon$ in the separation between the quarks in the diquark as illustrated in Fig. 5 one finds that the effective $q-q$ string tension is $(\sqrt{3} / 2) \sigma$ where $\sigma$ is the $q-\bar{q}$ string tension. Because in the perturbative regime, the $q-q$ attraction is also weaker than the $q-\bar{q}$ attraction, this time by a factor $\frac{1}{2}$, the conclusion is that for all distances one expects the $q-q$ attraction to be weaker. Thus a $q-q$ diquark should be somewhat larger than a $q-\bar{q}$ meson, which is what we find. Size measurements for the bad diquark, on the other hand, show neither scaling nor convergence to a plateau value. The large values obtained, often similar to our box size, corroborate the weakness of spatial correlations in this channel.

In conclusion, we have evaluated the mass splittings and density correlators of the complete set of diquark channels

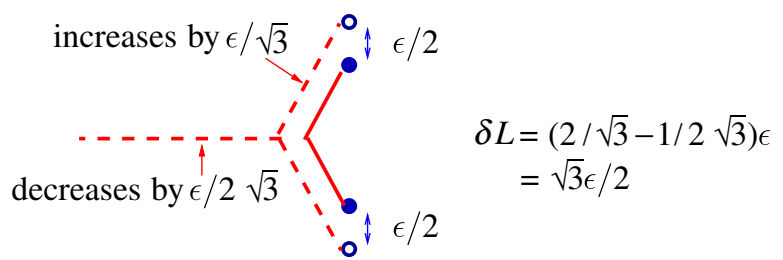

FIG. 5 (color online). String formation for three quarks, where one quark has been taken to the distant left. As a function of the separation between the other two quarks, the static potential rises with an effective string tension $\sigma_{\text {eff }}=\sigma(\sqrt{3} / 2)$.

created by local diquark fields. Both observables confirm the phenomenological expectation that QCD dynamics favors the formation of good diquarks, i.e., in the scalar positive parity channel. The characteristic size of this diquark, $\mathcal{O}(1) \mathrm{fm}$, is large but consistent with the scale $\mathcal{O}(200) \mathrm{MeV}$ of the attraction. Even a good diquark is a large object, which may limit its relevance to hadron structure. The positive parity vector channel is higher in energy by about $\frac{2}{3}$ the $\Delta$-nucleon mass splitting, and forms an even larger object. All the negative-parity channels have much higher energies.

We thank B. Orth, Th. Lippert, and K. Schilling [11] for providing the unquenched configurations. This work is supported in part by the EU Integrated Infrastructure Initiative Hadron Physics (I3HP) under Contract No. RII3-CT-2004-506078. The work of B.L. has been partially supported by the Royal Society.

[1] M. Ida and R. Kobayashi, Prog. Theor. Phys. 36, 846 (1966); for a review, see M. Anselmino et al., Rev. Mod. Phys. 65, 1199 (1993).

[2] R. L. Jaffe and F. Wilczek, Phys. Rev. Lett. 91, 232003 (2003).

[3] T. Nakano et al. (LEPS Collaboration), Phys. Rev. Lett. 91, 012002 (2003).

[4] S. Kabana, AIP Conf. Proc. 756, 195 (2005).

[5] R. L. Jaffe, Phys. Rep. 409, 1 (2005).

[6] M. Hess, F. Karsch, E. Laermann, and I. Wetzorke, Phys. Rev. D 58, 111502 (1998).

[7] C. Alexandrou, Ph. de Forcrand, and B. Lucini, Proc. Sci., LAT2005 (2006) 053.

[8] K. Orginos, Proc. Sci., LAT2005 (2006) 054.

[9] C. Alexandrou, Ph. de Forcrand, and A. Tsapalis, Phys. Rev. D 66, 094503 (2002).

[10] S. Necco and R. Sommer, Nucl. Phys. B622, 328 (2002).

[11] B. Orth, Th. Lippert, and K. Schilling, Phys. Rev. D 72, 014503 (2005).

[12] C. Alexandrou et al., Nucl. Phys. B414, 815 (1994).

[13] A. Hasenfratz and F. Knechtli, Phys. Rev. D 64, 034504 (2001).

[14] C. Alexandrou, G. Koutsou, J. W. Negele, and A. Tsapalis, Phys. Rev. D 74, 034508 (2006).

[15] Diquark mass splittings, measured in Ref. [8] for lighter quarks, tend to be larger than ours, with large uncertainties. 